\title{
An Zero-Flowrate-Switching (ZFS) Control Method Applied in a Digital Hydraulic System
}

\author{
Shuang Peng \\ School of Mechanical\&Mechatronic Engineering, Wuhan University of Technology, Wuhan, China \\ E-mail: shuang.peng@whut.edu.cn
}

\begin{abstract}
During past decades digital hydraulic technology has become the new trend in hydraulic area due to less consumed power, better controllability and larger tolerance of contaminations. In some digital hydraulic systems, PWM controller is applied to regulate the system flowrate. Thus pressure pulses and switching power loss resulted in by high frequent switching becomes the challenge of digital hydraulics. In this work, it aims to develop a control system where the digital valve always switches off at the moment when the value of the flowrate through the valve is around zero. In this paper a Zero-Flowrate-Swiching (ZFS) control system is presented in a application of a typical 2-valve digital hydraulic system. Operating principles and mathematical models are presented in the paper. According to the simulation results, the system with ZFS controller consumes $14.7 \%$ switching power loss compared to that of a HardSwitching (HS) control system. This gives a bright sight view that ZFS control method can be a potential solution for pressure pulses and switching power loss resulted by high frequent switching in digital hydraulic systems.
\end{abstract}

Keywords: Zero-flowrate-switching (ZFS), digital hydraulics, energy efficient.

\section{Introduction}

In 1988 Muto proposed a 2-way-solenoid-valves-system with PWM control for a hydraulic actuator [1]. In last decades digital hydraulic systems with different configurations have been more and more researched due to their potentials in higher efficiency performance and better characteristics of control methods. In 2000 Mattila published a two-valve actuator instead of a servo hydraulic system to actuate a manipulator arm with a load of $620 \mathrm{~kg}$ [2]. The proposed actuator have saved $15 \%$ of the total input energy. Peng compared the performance of four digital hydraulic systemsw with different configurations [3]. The results indicate that two-valve digital hydraulic systems show better energysaving performance than other systems.

However pressure pulses are the main challenge for the application of such digital hydraulic systems. When a digital valve switches off suddenly with high pressure and large flowrate, large pressure pulses would occur. During this process, energy would be wasted as switching loss and pressure pulses would make system response less accurate.

ZFS control method is to make valve switch off when the flowrate through the valve is reduced to zero[4-5]. In earlier work, ZFS control method is only modeled without cylinder load. Thus, dynamic capacitance from the cylinder is not included in old models. In this paper an improved ZeroFlowrate-Switching (ZFS) control method is applied in a two-valve digital hydraulic system. Capacitance from the load cylinder is included for better controllability.

This paper is organized as following sections. Section 2 introduces the operating principles and mathematic model of a ZFS control system applied in a typical 2-valve digital hydraulic system. Section 3 presents simulation results for the tested systems.

\section{A ZFS digital hydraulic system}

A typical 2-valve digital hydraulic system is concerned in this paper (shown in fig. 1). In this system valve 1 and valve 3 are used to control the directions of the flow. Two flow paths are presented by white/black arrows, respectively. In a ZFS control system, an accessory line (a resonant line) is mounted paralleled with valve 1 (shown in fig. 2). Smallamplitude waves in pressure output of regular pumps are used as the excitation of the resonant line. When the cylinder is retracting, the fluid goes as white arrows guide, valve 3 works at low pressure, switching loss is minor and pressure pulses can be ignored; when the cylinder is extending, the fluid flows following black arrows. During this process, valve 1 and valve 2 work at supply pressure. Large pressure pulses and considerable switching power loss happened here. Thus a ZFS control unit is designed here. A ZFS unit contains two parallel lines: one consists of valve 1; the other is accessory line which includes valve 2, a hydraulic capacitance and a hydraulic inductance. Valve 1 is the main control valve which is responsible for regulating 
flowrate for the cylinder when extending. When valve 1 is about to switch off, the accessory line starts to work until both valve 1 and valve 2 switch off at zero-flowrate moment.

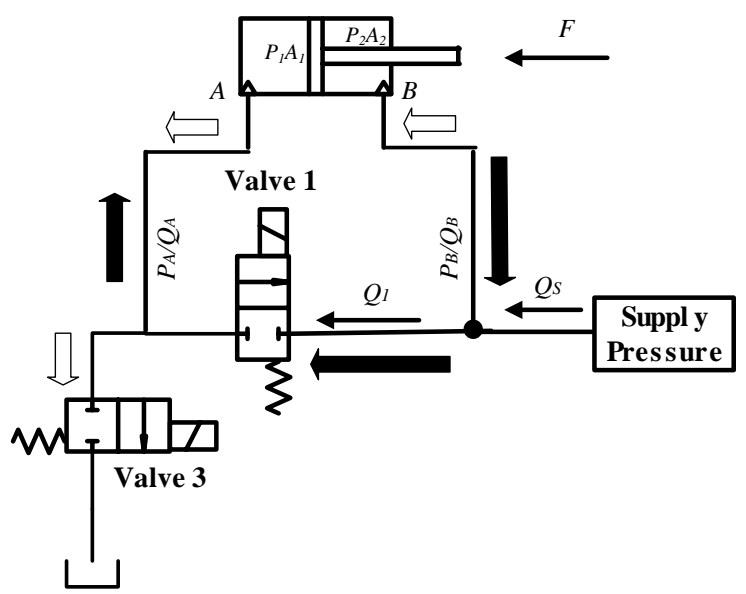

Figure 1 Schematic of a digital hydraulic system with two digital valves

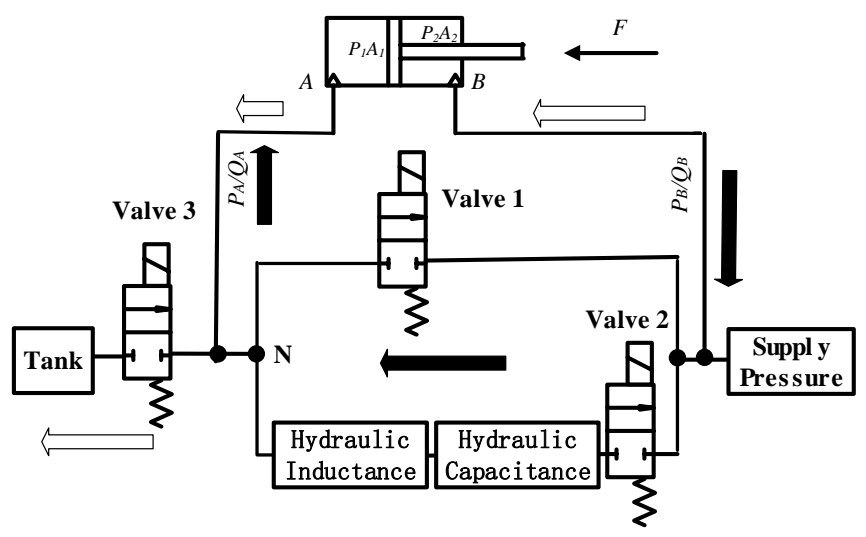

Figure 2: Schematic of a ZFS digital hydraulic system.

\subsection{Operating principles during extending process}

When the cylinder is extending, valve 1 works at high pressure, thus an accessory line is designed to make the valves switch off at zero-flowrate moments. The schematic of the system for extending motion is presented in fig. 3 .

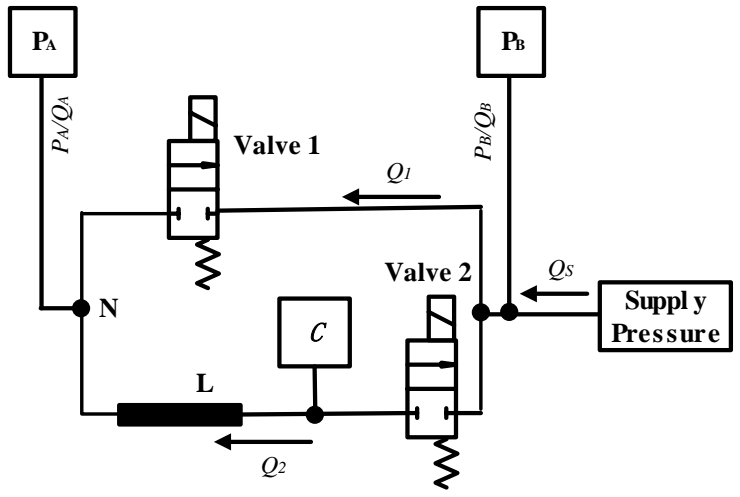

Figure 3: Schematic of a ZFS Unit.
The switching-off process is provided into four modes.

\section{Mode 1}

In mode 1 , valve 1 is on and valve 2 is off. Valve 1 functions the main control valve which regulates the load.

\section{Mode 2}

In mode 2 , valve 2 switches on while valve 1 still keeps on. Both the supplier and the RLC oscillator (shown in fig. 4) supply flow to the load.

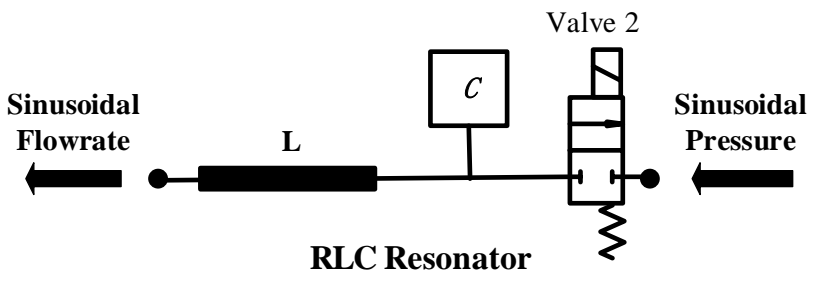

Figure 4: A Hydraulic RLC Resonator.

Mode 3

When the transient value of the sinusoidal flowrate out from the $R L C$ oscillator is equal to the value of required $Q_{A}$, the flow from both the supplier and rod-side of the cylinder is relived, the system switches to mode 3: valve 1 switches off at zero-flowrate moment, the accessory line supplies the load independently.

During this mode, the resonant flowrate is reduced gradually; After it oscillates back to zero, it flows to the opposite direction.

\section{Mode 4}

When its value is equal to $Q_{B}+Q_{\text {Smax }}$, the flowrate along accessory line is zero. Then valve 2 switches off, the system works in mode 4.

The state matrix of the valves are shown in Table 1.

Table 1 State matrix of the valves

\begin{tabular}{lll}
\hline Operating Modes & Valve 1 & Valve 2 \\
\hline Mode 1 & ON & OFF \\
Mode 2 & ON & ON \\
Mode 3 & OFF & ON \\
Mode 4 & OFF & OFF \\
\hline
\end{tabular}

\subsection{Mathematic models for extending process}

The supply pressure of the system is

$$
P_{S}=P_{S 0}+P_{S a} \sin (\omega t)
$$

The system works as a regular differential system in Mode 1

$$
Q_{S}=Q_{A}-Q_{B}
$$

In mode 2 and mode 3 , a resonator participates to actuate the system. Mathematics for mode 2 and mode 3 are presented 
to analyze the system performance. Following assumption are made for these models:

The orifice openings of valve 1 and valve 2 share the same shape and dimensions;

The pressure-vs-flowrate characteristic of valve 1 and valve 2 is linear within certain range of flowrate: $R=p / q$;

The flowrate-vs-opening-area characteristic of valve 1 and valve 2 is linear.

\section{Mode 2}

In this mode, both the main line and the accessory line are working (shown in fig. 5). The backflow of the cylinder and the flow from the supplier meet together, and then are split evenly into the main line and the accessory line. In the same time, the $R L C$ resonator starts to generate resonant flowrate $Q_{\text {res. }}$

$$
\begin{gathered}
Q_{1}=\frac{1}{2}\left(Q_{S}+Q_{B}\right) \\
Q_{2}=\frac{1}{2}\left(Q_{S}+Q_{B}\right)+Q_{r e s} \\
Q_{r e s}=\frac{P_{S a}}{R} \sin (\omega t)
\end{gathered}
$$

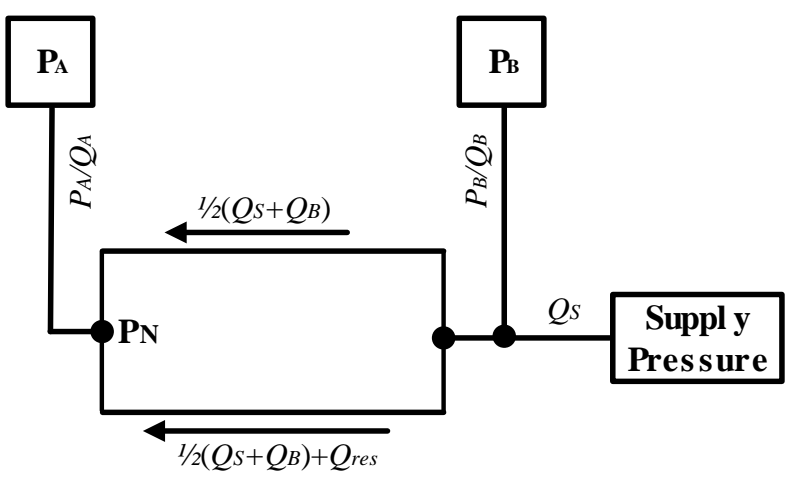

Figure 5: System schematic for mode 2

Right after valve 2 switches on, $Q_{S}$ is

$$
Q_{S}=Q_{A}-Q_{B}-Q_{\text {res }}
$$

Then $Q_{S}$ decxreases as $Q_{\text {res }}$ increases from zero(presented by red line in fig. 6).

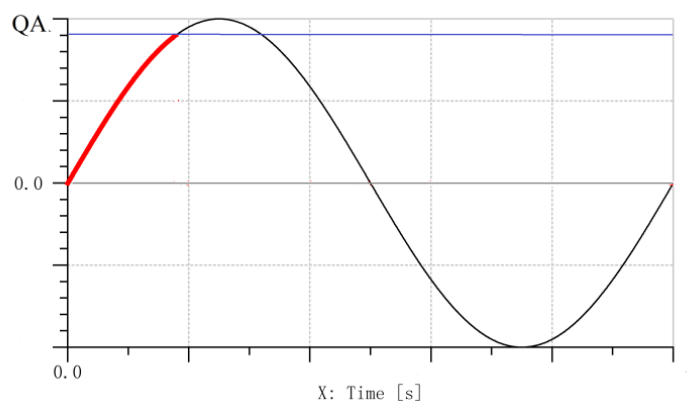

Figure 6: Resonant flowrate in mode 2

When the resonant flowrate $Q_{\text {res }}$ is up to $Q_{A}, Q_{S}$ is reduced to zero, QB is also relived by the relieve valve of the pump.
It is assumed that $Q_{r e s}\left(t^{*}\right)=Q_{A}$. At the moment $t=t^{*}$, the system behavior is complex. Here system behavior at the moments before and after $t=t^{*}$ is discussed.

\section{The system at the moment $t=t *_{-} 1 / \infty$}

When time is about to achieve $t^{*}, Q_{\text {res }}$ is about to achieve $Q_{A}$

$$
Q_{\text {res }}+\frac{1}{\infty}=Q_{A}
$$

Thus

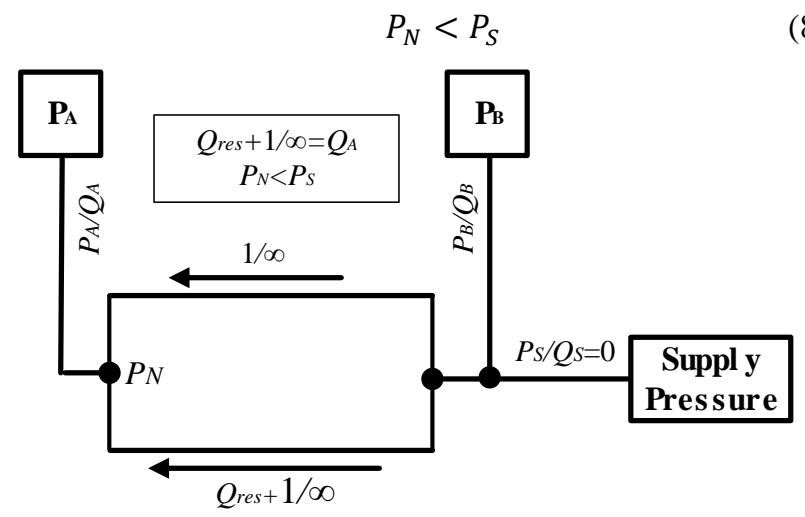

Figure 7: System schematic when $t=t^{*}-1 / \infty$

At this moment both lines are still working as presented in fig. 7 .

\section{The system at the moment $t=t^{*}+1 / \infty$}

When time is right after $t^{*}, Q_{\text {res }}$ is a little bit larger than $Q_{A}$, which is

$$
Q_{\text {res }}+\frac{1}{\infty}=Q_{B}+Q_{\text {Smax }}
$$

Then the actual flowrate to the rodless-side chamber is more then required $\left(Q_{A}\right)$, which results

$$
P_{N}>P_{S}
$$

The fluid cannot flow to the rodless-side chamber by the main line, thus

$$
\begin{gathered}
Q_{1}=0 \\
Q_{2}=Q_{B}+Q_{r e s}
\end{gathered}
$$

The direction of $Q_{1}$ is from node $\mathrm{N}$ to the power supplier. The schematic is presented in fig. 8 .

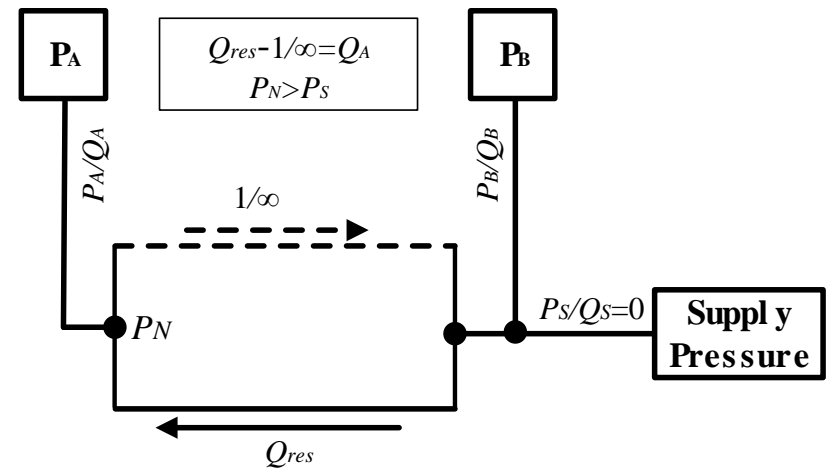

Figure 8: System schematic when $t=t^{*}+1 / \infty$ 


\section{Mode 3}

In mode 3 , the resonant flowrate $Q_{\text {res }}$ (shown by red line in fig. 9) keeps going up until it achieves the peak value; during this process, the pressure of $P_{A}$ is slightly boomed since valve 1 is switching off. After that $Q_{\text {res }}$ starts to fall down.

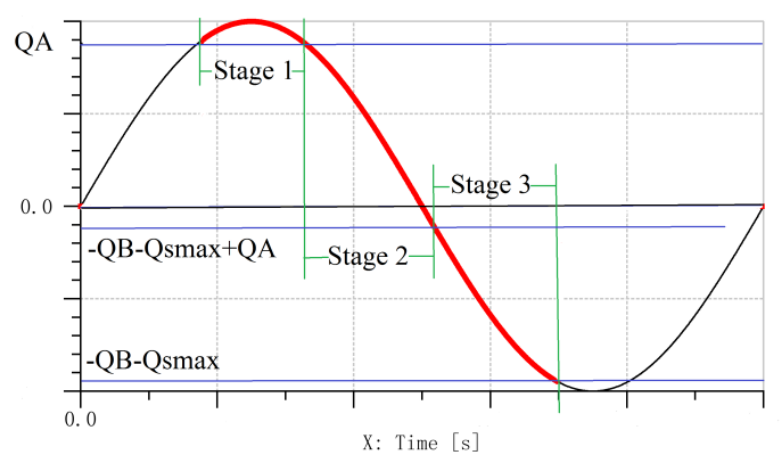

Figure 9: Resonant flowrate in mode 3

The schematic for mode 3 is presented in fig. 10.

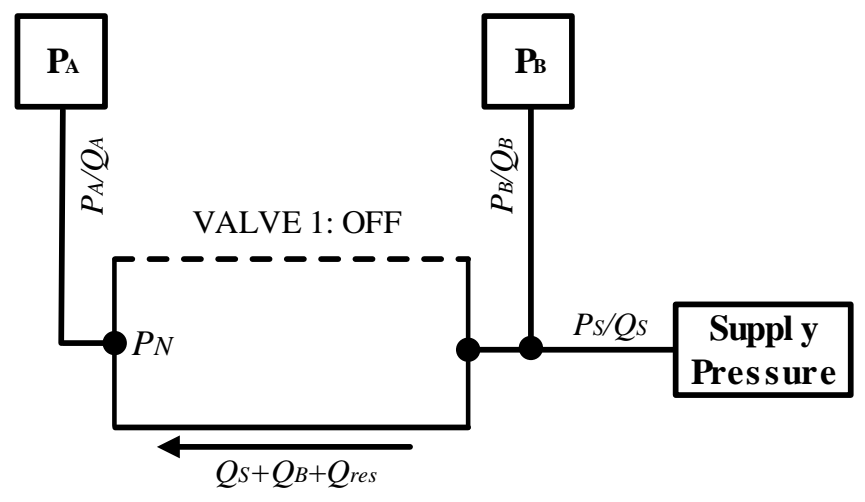

Figure 10: Resonant flowrate in mode 3

The system goes through three stages in mode 3.

\section{Stage 1}

Valve 1 is switching off, $Q_{B}$ and $Q_{S}$ stops supplying the system.

$$
\begin{gathered}
Q_{1}=C_{\text {orif }}\left(Q_{\text {res }}-Q_{A}\right) \\
Q_{2}=Q_{\text {res }}
\end{gathered}
$$

where

$$
C_{\text {orif }}=1-\frac{\Delta t}{T_{S}}
$$

Stage 2

Valve 1 is off, $Q_{\text {res }}$ is less than $Q_{A}$, thus part of $Q_{B}+Q_{S}$ helps supplying the system to make $Q_{2}$ equal to $Q_{A}$.

$$
\begin{aligned}
& Q_{1}=0 \\
& Q_{2}=Q_{A}
\end{aligned}
$$

At the end of stage 2

$$
Q_{\text {res }}=Q_{A}-Q_{B}-Q_{\text {Smax }}
$$

\section{Stage 3}

Valve 1 is off. The direction of Qres is from node $\mathrm{N}$ to the power source. As the value of Qres increases, all the flow of QB and QSmax is supplying the system, however, Q2 is still less than QA.

$$
\begin{gathered}
Q_{1}=0 \\
Q_{2}=Q_{\text {Smax }}+Q_{B}+Q_{\text {res }}
\end{gathered}
$$

At the end of stage 3

$$
Q_{\text {res }}=-\left(Q_{B}+Q_{\text {Smax }}\right)
$$

$Q_{2}$ turns to zero, valve 2 is ready to switch off.

\section{Mode 4}

Valve 1 is off. At the very beginning, valve 2 is switching off, thus Q2 is derived

$$
Q_{2}=C_{\text {orif }}\left(Q_{\text {Smax }}+Q_{B}-Q_{\text {res }}\right)
$$

\subsection{Switching power loss}

Theoretically, the fast switching valve works only in ON and OFF states, the switchging power loss is supposed to be zero. However, Physical valves are not idea ones. During the switching process, both flowrate and pressure difference exists, switching power loss is small but not zero.

During the switching process, switching power loss happens twice, for valve and valve 2 , respectively.

At the beginning of mode 3 , the switching power loss for valve 1 is derived as

$$
E_{\text {loss_ } 1}=\int_{\mathrm{t}}^{\mathrm{t}+T_{S}}\left(P_{S}(t)-P_{A}\right) C_{\text {orif }}\left(Q_{\text {Smax }}+Q_{B}-Q_{\text {res }}\right) d t
$$

At the beginning opf mode 4 , the switching power loss for valve 2 is derived as

$$
E_{\text {loss_ } 2}=\int_{\mathrm{t}}^{\mathrm{t}+T_{S}}\left(P_{S}(t)-P_{A}\right) C_{\text {orif }}\left(Q_{\text {Smax }}+Q_{B}-Q_{\text {res }}\right) d t
$$

\section{Simulation model and result analysis}

\subsection{Simulation model}

To analyze a ZFS control system, a supply pressure averaged at $20.5 \mathrm{MPa}$ with wave frequencies of $113 \mathrm{~Hz}$ is used as power source; the amplitudes for both waves are 2bar; the capacitance volume of the resonator is $0.8 \mathrm{~L}$. The closing time of the valve is assumed to be $0.002 \mathrm{~s}$.

The differential cylinder is actuated as shown in fig. 1 . The piston of the cylinder moves at constant speed. Since the switching-off process is very short, $P_{A}$ is assumed to be constant to simplify the model.

As contrast a system without accessory line is used to compare power assumption with the tested system. This system is also called Hard-Switching (HS) control system.

More details about the system parameter is presented in Table1. 
Table 2 System parameters

\begin{tabular}{ll}
\hline Operating Modes & Values \\
\hline$\rho$ & $860 \mathrm{~kg} / \mathrm{m}^{3}$ \\
$E$ & $14000 \mathrm{bar}$ \\
$V$ & $0.8 \mathrm{~L}$ \\
$D$ & $0.008 \mathrm{~m}$ \\
$l$ & $0.2 \mathrm{~m}$ \\
$f_{l}$ & $113 \mathrm{~Hz}$ \\
$P_{S a}$ & $2 \mathrm{bar}$ \\
$P_{S 0}$ & $205 \mathrm{bar}$ \\
$P_{A}$ & $190 \mathrm{bar}$ \\
$Q_{n o r m}$ & $20 \mathrm{~L} / \mathrm{min} @ 5 \mathrm{bar}$ \\
$Q_{A}$ & $7 \mathrm{~L} / \mathrm{min}$ \\
$Q_{B}$ & $2.195 \mathrm{~L} / \mathrm{min}$ \\
$Q_{S \max }$ & $4.805 \mathrm{~L} / \mathrm{min}$ \\
\hline
\end{tabular}

\section{2 result analysis}

Fig. 11 and fig. 12 presents an entire switching process for the two control systems. In a HS control system (Fig. 10), the valve linearly switch off, thus the flowrate decreases linearly. The transient power consumed over the valve goes up firstly and then goes down until the switching is finished. The peak power consumption is about $175 \mathrm{~W}$. The total switching power loss is $1570 \mathrm{~J}$.
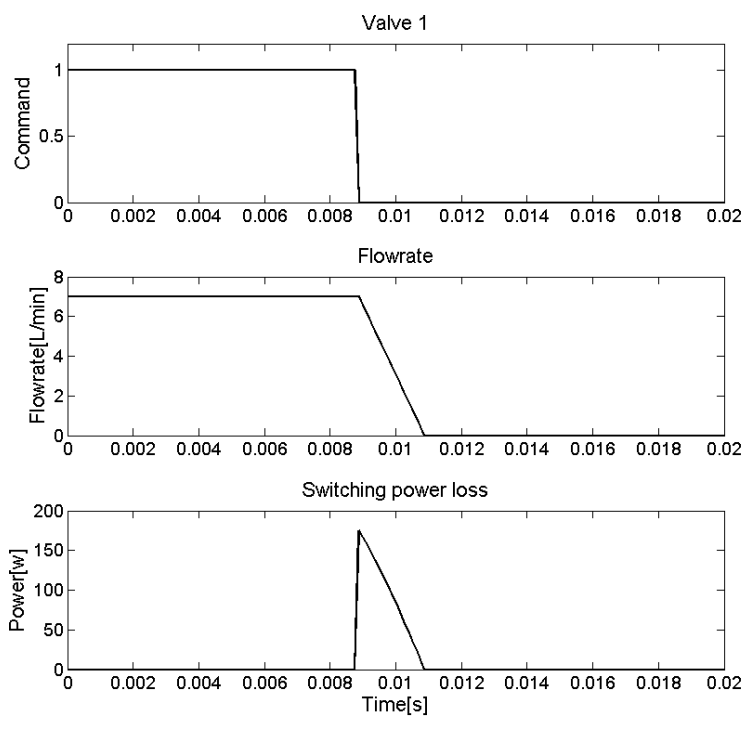

Figure 11: Simulation result for HS system

Fig. 12 presents the results for ZFS control system. at the beginning of the test, valve 1 (the control valve) is on and valve 2 (the resonant valve) is off; then valve 2 switches on and $Q_{2}$ goes up along the sinusoidal trajectory; correspondingly $Q_{l}$ decreases accordingly; soon $Q_{l}$ reaches zero. When $Q_{l}$ achieves zero-flowrate moment valve 1 is switched off. At the same time, $Q_{2}$ keeps going down until it achieves zero. Then valve 2 switches off. During the switching of valve 1 and valve 2 the power consumption happens since orifice flowrate exists. This is an entire zeroflowrate-switching control process. The peak power consumption is about $60 \mathrm{~W}$. The switching power loss consumed by ZFS system is about $230 \mathrm{~J}$ compared to $1570 \mathrm{~J}$ consumed by the counterpart. The result indicates that by introducing ZFS control method, the switching power loss is reduced to $14.7 \%$.
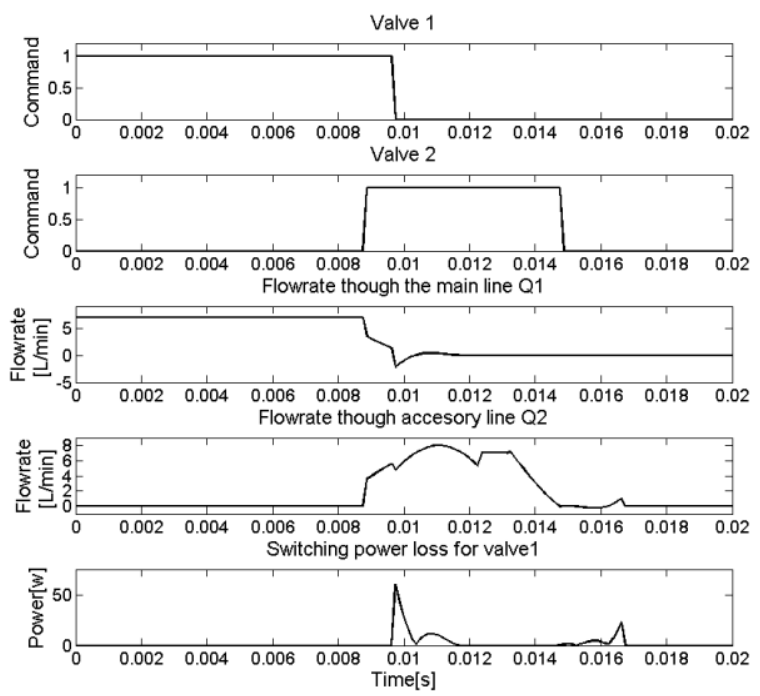

Figure 12: Simulation result for ZFS system

\section{Conclusions and future work}

This paper proposes a ZFS control method for a typical 2valve digital hydraulic system. The work focuses on switching power loss of digital valves. The result indicates that switching energy consumed by the valve in ZFS control system is about $14.7 \%$ compared to that in a HS control system. Since this method is to greatly reduce switching power loss, and theoretically remove switching pulse, it is a potential solution to improve the performance of digital hydraulic systems and other systems which need frequent switching.

In this paper, constant $P_{A}$ is applied to discuss the studied case. When the load is varied, the system response would change accordingly. Dynamic load with varied load force and changing movements will be discussed in future work.

\section{Acknowledgement}

This work is funded by State Key Laboratory of Fluid Power and Mechatronic System, the funding number is GZKF-201520, as well as Wuhan University of Technology, the funding number is 2017IVA013.

\section{Nomenclature}

\begin{tabular}{lll}
\hline Designation & Denotation & Unit \\
\hline$Q_{l}$ & Flowarte through valve 1 & $\mathrm{L} / \mathrm{min}$
\end{tabular}




\begin{tabular}{|c|c|c|}
\hline$Q_{2}$ & Flowarte through valve 2 & $\mathrm{~L} / \mathrm{min}$ \\
\hline$Q_{A}$ & Flowrate at rodless side & $\mathrm{L} / \mathrm{min}$ \\
\hline$Q_{B}$ & Flowrate at rod side & $\mathrm{L} / \mathrm{min}$ \\
\hline$Q_{S}$ & Flowrate from the supplier & $\mathrm{L} / \mathrm{min}$ \\
\hline$Q_{\operatorname{Smax}}$ & $\begin{array}{l}\text { Maximam flowrate from the } \\
\text { supplier }\end{array}$ & $\mathrm{L} / \mathrm{min}$ \\
\hline$Q_{\text {res }}$ & Resonant flowrate & $\mathrm{L} / \mathrm{min}$ \\
\hline$q$ & Flowrate & $\mathrm{L} / \mathrm{min}$ \\
\hline$P_{A}$ & Pressure in rodless-side chamber & Bar \\
\hline$P_{B}$ & Pressure in rod-side chamber & Bar \\
\hline$P_{N}$ & Pressure at node $\mathrm{N}$ & Bar \\
\hline$P_{S}$ & Supply pressure & Bar \\
\hline$P_{S O}$ & Average value of supply pressure & Bar \\
\hline$P_{S a}$ & Amplitude of the pressure waves & Bar \\
\hline$P^{*}$ & $\begin{array}{l}\text { Pressure generated by the various } \\
\text { volume of the rodless-side } \\
\text { chamber of the cylinder }\end{array}$ & Bar \\
\hline$P_{\text {loss } \_}$ & Switching power loss for valve 1 & $\mathrm{~W}$ \\
\hline$P_{\text {loss_ } 2}$ & Switching power loss for valve 2 & $\mathrm{~W}$ \\
\hline$p$ & Pressure & Bar \\
\hline$L$ & Coefficient of inductance & \\
\hline$C$ & Coefficient of capacitance & $\mathrm{m}^{3} / \mathrm{Pa}$ \\
\hline$C_{\text {orif }}$ & Orifice coeffecient & Null \\
\hline$E$ & Bulk modules of the fluid & Bar \\
\hline$F$ & Load force & $\mathrm{N}$ \\
\hline$R$ & $p / q$ & $\begin{array}{l}\mathrm{Bar} \bullet \\
\min / \mathrm{L}\end{array}$ \\
\hline$V_{\text {pipe }}$ & $\begin{array}{l}\text { volume of the pipe between the } \\
\text { node } \mathrm{N} \text { and the port of the } \\
\text { rodless-side chamber of the } \\
\text { cylinder }\end{array}$ & $\mathrm{m}^{3}$ \\
\hline$x$ & Position of the cylinder piston & $\mathrm{m}$ \\
\hline$A_{p i s}$ & Area of the cylinder piston & $\mathrm{m}^{2}$ \\
\hline$\omega$ & $\begin{array}{l}\text { Angular frequency of the supply } \\
\text { pressure waves }\end{array}$ & $\mathrm{Rad} / \mathrm{s}$ \\
\hline$t$ & Time & $\mathrm{s}$ \\
\hline$t^{*}$ & The end of mode 2 & $\mathrm{~s}$ \\
\hline$\Delta t$ & Time difference & $\mathrm{s}$ \\
\hline$T_{S}$ & Switching time & $\mathrm{s}$ \\
\hline
\end{tabular}

\section{References}

[1] T. Muto, H. Yamada, Y. Suematsu. PWM-Digital control of hydraulic actuator utilizing 2-way solenoid

valves. Jour. Jpn. Hydraulic \& Pheumatica Soc., (in Japanese), Vol. 14, No. 7, pp. 61 68.

[2] J. Mattila, and T. Virvalo. Energy-efficient motion control of a hydraulic manipulator. Proc. of the International Conference on Robotics \& Automation, Sanfrancisco, CA, USA, 2000.

[3] S. Peng, D. Branson, E. Guglielmino, and D. G. Caldwell. Simulated performance assement of different digital hydraulic configurations for use on the HyQ leg. Proc. of International Conference on Robotics and Biomimetics, Guangzhou, China, 2012.

[4] S. Peng. The concept of a zero-flowrate-switching controller. Proc. of Eighth Workshop on Digital Fluid Power. Tampere, Finland, 2016.

[5] S. Peng. A zero-flowrate-switching (ZFS) control system applied in a simple hydraulic line. Proc. of $9^{\text {th }}$ International Conference on Fluid Power Transmission and Control, Hangzhou, China, 2017. 